\title{
Consumo de alcohol y drogas en estudiantes de Pachuca, Hidalgo*
}

\author{
Estela Rojas-Guiot, Lic. en Psic., ${ }^{(1)}$ Clara Fleiz-Bautista, Lic. en Psic., ${ }^{(1)}$ \\ María Elena Medina-Mora Icaza, Dra. en Psic., ${ }^{(2)}$ Ma. de los Angeles Morón, Lic. en T.S. ${ }^{(3)}$ \\ Melanie Domenech-Rodríguez, M. en Psic.(4)
}

\begin{abstract}
Rojas-Guiot E, Fleiz-Bautista C, Medina-Mora Icaza ME, Morón MA, Domenech-Rodríguez $M$.

Consumo de alcohol y drogas en estudiantes de Pachuca, Hidalgo. Salud Publica Mex 1999;41:297-308.
\end{abstract}

\section{Resumen}

Objetivo.D eterminar la prevalencia del consumo de drogas y bebidas alcohólicas entre estudiantes, así como la relación de este consumo con variables sociodemográficas, tiempo libre, actos antiso ciales, no rmas y conflictos familiares, entre otras. Material y métodos. Los datos se derivan de una encuesta representativa de 1929 estudiantes de enseñanza media y media superior de la ciudad de Pachuca, estado de Hidalgo, México, levantada en 1996, de los cuales $44.9 \%$ son hombres y $52.5 \%$ mujeres, con una media de edad de 14 años. Se utilizó un cuestionario autoaplicable, que incluye indicadores de consumo de drogas y alcohol elaborados por algunos países, entre ellos México, con la 0 rganización Mundial de la Salud. Resultados. El $47.9 \%$ ha probado bebidas alcohólicas; $12.6 \%$ bebieron cantidades considerables - cinco copas o más por ocasión- durante el mes anterior a la encuesta. Las bebidas preferidas son la cerveza y los coolers; el alcohol lo compraron principalmente en tiendas donde no les piden identificación y lo consumen en su casa 0 en la de otras personas. El $5.1 \%$ ha consumido drogas, sobre todo inhalables, mariguana y tranquilizantes. U na cifra mayor de varones consume drogas ilegales, y las mujeres, medicamentos sin prescripción. Un número superior de ho mbres toma más cantidades de alcohol y consume dro gas,
Rojas-Guiot E, Fleiz-Bautista C,

Medina-Mora Icaza ME, Morón MA,

Domenech-Rodríguez $M$.

Alcohol and drug consumption among

students from Pachuca, Hidalgo.

Salud Publica Mex 1999;41:297-308.

\begin{abstract}
A bstract
Objective.To determine the prevalence of alcohol and drug consumption and its relationship to sociodemographic variables, leisure activities, antisocial behavior, family norms and conflicts, among others. Material and methods. D ata derive from a representative survey of 1929 students of junior high and high school, conducted in 1996 in the city of Pachuca, Hidalgo, Mexico. $\mathrm{O}$ these, $44.9 \%$ were boys and $52.5 \%$ were girls; mean age was 14 . A self-applied questionnaire, prepared by theW HO together with some countries, among them Mexico, was completed by the studied subjects, and included indicators of alcohol and drug consumption. Results $0 \mathrm{f}$ the total sample, $47.9 \%$ had tried alcohol, and $12.6 \%$ had drunk large quantities -5 drinks or more per sitting- during the month previous to the survey. Preferred drinks are beer and "coolers", which they buy at shops where no identification is required and drink at home or at friend's homes.W ith respect to drugs, $5.1 \%$ had tried illegal or medical drugs without prescription, in particular inhalants, marihuana and tranquilizers. More boys consumed illegal drugs, and more girls medical drugs without prescription. Boys, who are also older, more frequently consumed alcohol and drugs and were more often employed during the previous year at part-time jobs. High alcohol level and drug
\end{abstract}

* Esta investigación fue financiada por el Consejo Nacional de Ciencia y Tecnología, clave M0148-H 9602, y por el Instituto Mexicano de Psiquiatría, proyecto N 0.4270 , y subestudio en escuelas proyecto N 0.4274 P.

(1) Investigadoras de la División de Investigaciones Epidemiológicas y Sociales, Instituto Mexicano de Psiquiatría (IMP), México.

(2) Jefa de la División de Investigaciones Epidemiológicas y Sociales, IMP, México.

(3) Secretaria Técnica del Consejo Estatal contra las Adicciones,Secretaría de Salud, Estado de Hidalgo, México.

(4) Investigadora del Tri-Ethnic Center for Prevention Research, Colorado State University, Estados Unidos de América.

Fecha de recibido: 11 de noviembre de 1998 • Fecha de aprobado: 7 de abril de 1998

Solicitud de sobretiros: Lic. Estela Rojas Guiot. Instituto Mexicano de Psiquiatría. Calzada México-X ochimilco 101, colonia San Lorenzo Huipulco, Delegación Tlalpan, 14370 México, D.F., México.

Correo electrónico: rojasgm@ imp.edu.mx 
tienen mayor edad y trabajaron de medio tiempo durante el año anterior a la encuesta. Los consumidores de drogas y de altas cantidades de alcohol se distinguieron porque un número considerable informó que se aburría en su tiempo libre, se va a beber con sus amigos o ha cometido actos antisociales. Respecto a la familia, manifiestan cumplir menos con las normas parentales y muestran menor interes en hacerlo. Asimismo, un porcentaje más elevado informó que sus padres pelean con frecuencia, han pedido ayuda por esa razón y han intentado separarse. Conclusiones Los estudiantes que beben cantidades elevadas de alcohol y los consumidores de otras drogas, a diferencia de lo que sucede con los abstemios, presentaron más problemas al destinar su tiempo libre a actividades como irse a beber con amigos, exhibir más conductas antisociales y tener un distanciamiento de la familia que se refleja en la baja participación en actividades compartidas, en el desinterés por cumplir con sus normas y en la percepción de conflictos al interior de la misma.

Palabras clave: consumo de bebidas alcohólicas; abuso de sustancias; estudiantes; México consumers were characterized by their frequent report of being bored in their free time, drinking with friends and enrolling in antisocial behavior.W ith respect to family nor$\mathrm{ms}$, they follow them less and show less interest in doing so An elevated percentage informed that their parents fight frequently, that they have sought help for this reason and have intended separation. Conclusions Groups who drink more alcohol and use other drugs, in contrast with nonusers, presented more behavioral problems, more outdoors activities that included drinking with friends, more antiso cial behavior, had a distant relationship with their families sharing few activities with them, an showed little inter est in following family rules and perceiving conflicts within their families.

Key words: alcohol consumption; substance abuse; students Mexico
$\mathrm{D}$ Ía con día se hace más evidente el aumento en los índices de consumo de drogas entre la población juvenil pues se trata de una situación que, por los problemas que ocasiona tanto desde el punto de vista personal como desde el comunitario, se refleja en los diferentes ámbitos sociales. Sin embargo, las cifras de consumidores de alcohol y drogas y las consecuencias para la salud no presentan una situación uniforme en el país, por lo que es necesario estudiar su distribución por entidad; en este trabajo se aborda el fenómeno en la comunidad estudiantil de la ciudad de Pachuca, Hidalgo.

En la Encuesta Nacional de Adicciones realizada en 1988, se encontró que $51 \%$ de la población entre los 18 y 65 años de edad consumía alcohol; 82\% eran hombres, y 44\%, mujeres; ${ }^{1}$ y en el grupo de 15 a 18 años $46.8 \%$ bebían alcohol. ${ }^{2}$ De todas las regiones en que se dividió al país, la centro (formada por las entidades de Hidalgo, Guanajuato, Estado de México, Morelos, Puebla, Querétaro y Tlaxcala) fue la que tuvo el porcentaje más alto de personas con síndrome de dependencia al alcohol. ${ }^{3}$ En esta misma zona del país se han observado las cifras más altas de cirrosis hepática y el consumo de bebidas alcohólicas es uno de los principales factores asociados a dicha enfermedad. ${ }^{4}$ En 1995 las tasas de mortalidad por cirrosis hepática en el estado de Hidalgo fueron las más elevadas en el país. ${ }^{5}$

Según la misma encuesta de 1988, 4.7\% de la población del país había consumido otras drogas diferentes al alcohol o al tabaco, y los jóvenes (entre 12 y 34 años) presentaron la cifra más alta $8.5 \%{ }^{6,7}$ Se observa- ron tendencias similares en la segunda Encuesta Nacional de Adicciones levantada en 1993.*8

En el caso concreto de la población de estudiantes, el alcohol también es la droga que ocupa el primer lugar de consumo. ${ }^{9-11}$ En la Encuesta Nacional sobre el Uso de Drogas en la Comunidad Escolar realizada en 1991 con muestras representativas de las 32 entidades del país, 49.6\% había probado bebidas alcohólicas; 3\% declararó beber cinco o más copas una o dos veces por semana, 40 hombres y 10 mujeres de cada $1000,{ }^{10} \mathrm{y}$ en el estado de Hidalgo 1.4\% de los estudiantes lo hacían; $2.1 \%$ eran varones y el $0.5 \%$ mujeres. ${ }^{\ddagger}$

En esta investigación nacional de 1991, 14.7\% de los alumnos del país tomaron alcohol en el mes previo a la encuesta, y en el estado de Hidalgo la cifra fue ligeramente inferior, esto es, $10.1 \%$. Respecto al consumo de drogas ilegales y médicas, los datos de esta misma encuesta indican que Hidalgo ocupó el tercer lugar (10.4\%) en el número de consumidores, después de Baja California (12.9\%) y del Distrito Federal (11.2\%), comparadas con todas las demás entidades del país.

\footnotetext{
* Dirección General de Epidemiología-Secretaría de Salud. Encuesta Nacional de Adicciones. Tomo Alcohol. México, D.F.: DGE, 1993. Documento no publicado.

₹ Instituto Mexicano de Psiquiatría-Secretaría de Salud/Dirección General de Educación Extraescolar-Secretaría de Educación Pública. Encuesta Nacional del Uso de Drogas en la Comunidad Escolar. Tomo Hidalgo. México, D.F., 1991. Documento no publicado.
} 
La necesidad de conocer la evolución local del problema y abordar en forma específica a la población estudiantil de enseñanza media y media superior es importante, ya que de acuerdo con De la Fuente ${ }^{12}$ y Medina-Mora, ${ }^{13}$ la mayoría de las personas empiezan a consumir sustancias psicoactivas en la adolescencia, y entre los factores de riesgo que tienen estos jóvenes para consumirlas se han identificado los siguientes: pertenecer al sexo masculino, asociarse con consumidores de drogas, ausentarse frecuentemente de la escuela, falta de compromiso con la misma, percepción de falta de afecto parental, poco apego y supervisión de los padres, prácticas de manejo familiar incongruente, padres que consumen drogas o alcohol, búsqueda de sensaciones nuevas, y problemas emocionales y conductuales, entre otros.

Así, a mayor cantidad de factores de riesgo a los que esté expuesto el individuo, más probabilidades habrá de que consuma drogas. De esta forma, el entorno social en el que se ubica el fenómeno del consumo de drogas, como la familia, las creencias, las actitudes, las normas, las costumbres y, en general, el medio en el cual se desenvuelve el sujeto, son variables que ayudan a comprender y a explicar esta problemática.

De estos múltiples factores, se ha elegido estudiar la relación entre el consumo de alcohol y otras drogas con el uso del tiempo libre, la influencia de la familia $\mathrm{y}$, de los pares en dos dimensiones, la parte normativa de la conducta de sus miembros y la influencia de los conflictos familiares en la utilización de sustancias para enfrentar problemas; también se aborda la relación entre el consumo de alcohol y drogas, y los problemas de conducta.

En particular, la influencia familiar desempeña un papel relevante como agente socializador en la vida del individuo, propiciando un desarrollo sano y maduro, o bien, inhibiendo o desviando su crecimiento. ${ }^{14}$ Es decir, puede ubicar al joven en riesgo o protegerlo de involucrarse en conductas desviadas tales como el consumo y el abuso en el consumo de sustancias o la incurrencia en actos antisociales. En general, los datos que se han encontrado en investigaciones realizadas en poblaciones estudiantiles señalan que existe una correlación positiva entre el consumo de drogas y la conducta antisocial, de tal manera que se puede afirmar que a mayor índice de consumo, mayor probabilidad de que se presenten también problemas de conducta. ${ }^{15-18}$

Aunque la influencia de la familia disminuye progresivamente en la medida en que el menor pasa de la niñez a la adolescencia, sigue siendo importante en el establecimiento de las normas que moldean la conducta de consumo de los adolescentes; por otra parte, la presencia de conflictos en la familia, aumenta el riesgo de que los adolescentes consuman drogas. La familia influye asimismo en la elección que hacen los adolescentes de sus amigos, quienes durante esta época desempeñan un papel fundamental en la conducta de los menores. En una investigación con estudiantes del Distrito Federal ${ }^{19}$ se encontró que, si bien la familia no determinaba en forma directa el consumo de sustancias por parte del adolescente, sí tenía una gran influencia para que éste se involucrara con amistades que las consumían. Es así como el hecho de que un alumno tenga amigos que son consumidores le hace correr un riesgo 1.22 veces más elevado de convertirse, a su vez, en consumidor de drogas. Asimismo, en una encuesta entre estudiantes que se aplicó en Estados Unidos de América (EUA), ${ }^{20}$ se encontró que el consumo de drogas entre los pares era el predictor principal del consumo de alcohol entre los adolescentes. Sin embargo, las normas que tenían los padres también resultaron ser significativas en la predicción del abuso en el consumo de alcohol entre estos jóvenes.

Por otro lado, si los familiares (padre, madre, hermanos) ingieren drogas, existe un riesgo 1.10 veces mayor de que el joven, a su vez, las consuma. ${ }^{19}$ En una encuesta nacional realizada en EUA, ${ }^{21}$ los autores encontraron que, en contraste con los no consumidores, los familiares de los consumidores de drogas, consumían drogas y alcohol en exceso, tenían un ambiente familiar inestable y desorganizado, así como un número más alto de conflictos intrafamiliares, separaciones y divorcios. En Suecia, Muetzell ${ }^{22}$ realizó un estudio de seguimiento durante tres años con adolescentes que consumían alcohol y drogas; sus resultados mostraron que, en contraste con los controles, un mayor número de los sujetos que combinaban el consumo de alcohol con otras drogas, crecieron en familias que consumían drogas, además de que sus hogares estaban desintegrados.

En términos de interacción familiar, si el adolescente percibe un ambiente caótico, poco estable $\mathrm{e}^{23} \mathrm{y}$ punitivo, así como conflictos generados al interior de la familia, es más probable que busque, por medio de los amigos, situaciones que le ayuden a resolver y enfrentar estos problemas, además de los propios de la adolescencia. Esta búsqueda de soluciones lo pueden conducir a consumir alcohol o drogas ${ }^{24} \mathrm{y}$, en general, a exhibir conductas que impliquen un riesgo constante para su seguridad física y emocional. ${ }^{25}$ En un estudio que realizaron con jóvenes consumidores y no consumidores de drogas y alcohol, Maltzman y Schweiger ${ }^{26}$ observaron que los usuarios tenían relaciones familiares más disfuncionales, debido a que existía menos cohesión entre los miembros y una menor participación en actividades sociales, culturales y recreativas, satis- 
factorias y mutuamente compartidas. Los autores concluyeron que una característica de estas familias es la falta de apoyo emocional entre sus miembros.

Respecto a la relación entre la familia y la escuela, Pons y colaboradores ${ }^{27}$ realizaron una investigación con estudiantes españoles, y encontraron que los que han consumido drogas se sentían más desadaptados en su medio familiar y escolar que aquellos que no eran consumidores.

Finalmente, el tipo de actividades que desarrolla el menor influye en la conducta de consumo. Diferentes autores como Furlong y Cartmel ${ }^{28}$ han postulado que, en épocas de cambio social como la actual, en la medida en que las ligas familiares y la afiliación a la clase social pierden importancia, los estilos de vida, incluidas las actividades en que se involucra el menor en su tiempo libre y que forman parte importante de la cultura juvenil del momento, son centrales en el proceso de construcción de la identidad.

Las estrategias cada vez más complejas y sofisticadas del mercado tienen un impacto importante en los estilos de vida y en los patrones de consumo que tienden a simbolizar la identificación cultural. El incremento en los años que permanecen los jóvenes en la escuela y la ausencia de oportunidades de trabajo, han dado lugar a que pasen mucho más tiempo en un estado de semidependencia y en la compañía de sus pares, factor que, a su vez, ha ejercido una influencia importante en los estilos de vida y en los productos con los que se identifican. No es de extrañar entonces, que la estrategia de la industria alcoholera en la búsqueda de nuevos mercados entre los jóvenes haya impactado sus estilos de vida normalizando el consumo de bebidas alcohólicas.

Dado que no todos los menores de edad tienen un inicio temprano en el consumo, ni adoptan prácticas propias de la población masculina de edad media, esto es, el asociar las ocasiones de consumo con ocasiones de embriaguez ingiriendo grandes cantidades cuando beben, cabe preguntarse si existen variaciones en las actividades adoptadas durante el tiempo libre entre los estudiantes con diferentes tipos de patrones de consumo, con el fin de orientar los programas de prevención dirigidos a este grupo.

Tomando en cuenta todos estos aspectos, en 1996 se levantó esta encuesta entre estudiantes que formó parte de una investigación muy amplia realizada en diferentes poblaciones (adultos y jóvenes en hogares, personas que acuden a solicitar ayuda en hospitales, en Ministerios Públicos, así como al personal y clientes de una discoteca) de la ciudad de Pachuca, estado de Hidalgo, México. Su propósito principal era estudiar los factores de riesgo asociados al abuso en el consumo de bebidas alcohólicas y otras drogas, así como proponer medidas de intervención y hacer una evaluación de su impacto en la población.

Los principales objetivos del presente trabajo son:

- Dar a conocer las prevalencias del consumo de alcohol y de otras drogas.

- Identificar los conflictos, las normas familiares y las percepciones del consumo entre los familiares y amigos.

- Conocer los problemas, las razones para beber y los actos antisociales más frecuentemente asociados al consumo.

- Comparar estas variables entre los grupos de usuarios y no usuarios de alcohol y drogas.

\section{Material y métodos}

Se utilizaron los registros oficiales del Instituto Hidalguense de Educación, de todas las escuelas secundarias y preparatorias tanto públicas como privadas que había en la ciudad de Pachuca, durante el ciclo escolar 19961997, como marco muestral, con el objetivo de seleccionar una muestra representativa de los estudiantes de esa ciudad. La unidad de análisis sobre la que se obtuvo información fueron los estudiantes de esos dos niveles de estudio.

El cálculo para el tamaño de la muestra se hizo con base en los datos obtenidos en las anteriores investigaciones con estudiantes que han efectuado, desde hace dos décadas, la Secretaría de Educación Pública y el Instituto Mexicano de Psiquiatría, en particular en las levantadas en $1991,{ }^{*}, 10$ y en 1993., 29

Para determinar el tamaño de la muestra y estimar las prevalencias de consumo y abuso en el consumo, se consideró el índice de $2.6 \%$, de abuso en el consumo de bebidas alcohólicas (cinco copas o más), obtenido en las encuestas anteriores como la variable de interés. Se estimó una tasa de ausentismo de $20 \%$, que incluye la ausencia de los alumnos por diferentes causas en un día dado, una confianza de $95 \%$ y un error absoluto de 1\%. La muestra estimada fue de 2100 alumnos; de acuerdo con los datos del marco muestral, se calculó un promedio de 35 alumnos por cada grupo, lo cual dio como

\footnotetext{
* Pérez-Heredia R. Encuesta Nacional del Uso de Drogas en la Comunidad Escolar 1991. Documento metodológico. México, D.F.: Instituto Mexicano de Psiquiatría / Dirección General de Educación Extraescolar, 1991. Documento no publicado.

* Pérez-Heredia, R. Encuesta sobre las Tendencias del Uso de Drogas en la Comunidad Escolar del Distrito Federal 1993. Documento metodológico. Diseño de muestra. México, D.F.: Instituto Mexicano de Pediatría, 1993. Documento no publicado.
} 
resultado una estimación de un total de 60 grupos a seleccionar.

El diseño de la muestra fue autoponderado tanto para grupos como para alumnos, con la finalidad de facilitar el proceso de estimación y el procesamiento de la información.

El muestreo se realizó en dos etapas: en primer término, un experto llevó a cabo el cálculo para la selección de las escuelas, por lo que calculó un intervalo de selección uniforme para cada nivel de estudio; en cada uno se realizó un acumulado de grupos y, de acuerdo con el intervalo, se efectuó la selección de escuelas. Dentro de cada nivel educativo se inició con un nuevo arranque aleatorio para asegurar una selección independiente en cada uno de ellos, el cual se obtuvo al azar entre el 0 y el intervalo de selección calculado.

En la segunda etapa, al llegar a cada plantel se aplicó un formato denominado "Hoja de selección", mismo que contiene todos los datos de cada escuela seleccionada como dirección, turno y número de grupos; tiene dos columnas, en una se marca y posteriormente se cubre el (los) grupo(s) a los que se les administrará el cuestionario. Esta selección de grupos la hicieron los investigadores responsables previamente en gabinete, para evitar posibles errores en la selección. Lo anterior se logró al elegir un número aleatorio menor o igual al total de grupos proporcionados por el marco de muestreo de cada escuela seleccionada; al llegar a cada plantel, el encuestador elaboraba un listado, en la otra columna del formato, de los grupos existentes aplicaba los cuestionarios al (los) grupo(s) previamente marcado(s).

Se obtuvo una muestra de 1929 alumnos; se seleccionaron 23 escuelas secundarias y siete preparatorias, y como en algunas escuelas resultaron seleccionados más de un grupo, se obtuvieron en total 42 grupos en secundaria con 1367 alumnos y 19 grupos en preparatoria con 559 alumnos.

El procedimiento que se siguió para el levantamiento de la encuesta fue el siguiente: los encargados de aplicar los cuestionarios eran pasantes y profesionistas de carreras de ciencias sociales con experiencia en la aplicación de encuestas, a quienes se instruyó durante varios días para familiarizarlos con el cuestionario y con la logística de la encuesta; los investigadores responsables supervisaron todos los detalles del levantamiento.

Al iniciar la aplicación en cada uno de los grupos, los encuestadores explicaban a los alumnos que su participación era voluntaria, que la información que proporcionarían era confidencial y anónima, y que los datos se presentarían en forma de un resumen estadístico.
El cuestionario empleado es autoaplicable, está precodificado y contiene 158 preguntas cerradas así como indicadores que han sido elaborados por varios países, entre ellos México, conjuntamente con la Organización Mundial de la Salud. Este instrumento ha sido utilizado en diferentes encuestas con esta población, en las cuales se ha probado su validez y confiabilidad. ${ }^{30}$

Las escalas de interés para este trabajo son las siguientes:

- Datos sociodemográficos. En esta sección se incluyen preguntas como sexo, edad, ocupación y estudios en el año anterior a la encuesta.

- Consumo de sustancias. Esta sección tiene como objetivo estimar la prevalencia (alguna vez en la vida y en los últimos 30 días) de las siguientes drogas: anfetaminas, tranquilizantes, inhalables, mariguana, cocaína y alucinógenos.

- Consumo de bebidas alcohólicas. Aquí se obtiene la prevalencia del consumo de alcohol (alguna vez en la vida y en los últimos 30 días). La cantidad y frecuencia con la que beben, el tipo de bebidas que consumen, los lugares donde beben y donde obtienen el alcohol; los problemas relacionados con este consumo, el consumo de alcohol en familiares y amigos, y las razones para beber alcohol.

- Escala de tiempo libre. Permite evaluar las actividades que el adolescente suele realizar cuando no asiste a la escuela o al trabajo.

- Escala de actos antisociales. Está conformada por indicadores que miden la ocurrencia de conductas inadecuadas que hayan presentado los adolescentes en los últimos 12 meses.

- Seccion de conflictos familiares. Contiene preguntas acerca de los conflictos entre los padres y/o los adultos de la casa, si los alumnos los consideran graves, si han solicitado ayuda y si el consumo de alcohol ha ejercido alguna influencia sobre los mismos.

- Escala de normas familiares. Contiene reactivos acerca de las reglas familiares respecto al consumo de bebidas alcohólicas y si al adolescente le interesa cumplir con estas normas.

Para el análisis de los datos se utilizaron las pruebas estadísticas $x^{2}$, Kruskall Wallis y U de Mann Whitney, con la finalidad de detectar si había diferencias entre los estudiantes abstemios, los bebedores y los consumidores de otras drogas. Estos análisis se realizaron con el paquete SPSS para Windows, versión 7.0.

El patrón de consumo de bebidas alcohólicas se dividió en las siguientes categorías: 
- No bebedores: son los estudiantes que nunca en su vida han bebido alcohol.

- Beben menos de cinco copas: incluye a los que beben pero no lo hicieron en el último mes, y a los que bebieron entre una y cuatro copas en ese lapso.

- Beben cinco copas o más por lo menos una vez al mes: abarca a los que tomaron esta cantidad por ocasión de consumo, en el mes anterior.

Respecto del patrón de consumo de drogas, se distinguieron dos categorías:

- No usuarios: se refiere a los que nunca han consumido ninguna de las drogas investigadas.

- Usuarios: son los estudiantes que han consumido drogas alguna vez en su vida.

\section{Resultados}

\section{Datos sociodemográficos}

La proporción por sexo es muy similar: $44.9 \%$ son hombres y $52.5 \%$ son mujeres. Cerca de $80 \%$ de la muestra tiene entre 12 y 15 años, con un rango de 10 a 22 años y una media de 14 años. La mayoría estudió y no trabajó en el año anterior a la encuesta (85\%). Sin embargo, $12.3 \%$ tuvieron un empleo de medio tiempo o de tiempo completo y $15.8 \%$ estudió medio tiempo o no asistió a la escuela.

\section{Alcohol}

El 47.9\% ha probado bebidas alcohólicas alguna vez en su vida, de los cuales $50.6 \%$ son hombres, y $45.9 \%$, mujeres. Respecto al consumo en el mes anterior, 20.9\% bebió alcohol, y $12.6 \%$ lo hizo en altas cantidades, esto es, cinco copas o más por ocasión. De ellos, una cifra superior son hombres, tienen 18 años o más, no estudiaron y / o trabajaron medio tiempo en el año anterior a la realización de la encuesta (cuadro I).

Las bebidas preferidas de esta población son: la cerveza (30.5\%) y los coolers (29.9\%); en segundo lugar están los vinos de mesa (25.3\%) y los destilados (24.7\%). Cabe mencionar que las bebidas que consumen en mayores cantidades (cinco copas o más por ocasión) son la cerveza $(5.4 \%)$ y los destilados $(4.4 \%)$.

Los principales lugares en donde obtienen las bebidas alcohólicas son: "en una tienda sin pedirles identificación" (15.5\%); "en su casa con permiso de sus padres" (14.2\%); "sin permiso de ellos" (7.6\%); "con un amigo de menos de 18 años" (7.7\%), y en "venta clandestina" (4.5\%), es decir, en tiendas que no tienen autorización para vender bebidas alcohólicas, o que lo hacen fuera de su horario normal. Generalmente, beben en su propia casa $(20.6 \%)$ o en la de otra persona (12.5\%), en áreas abiertas como parques o calles $(10.1 \%)$, en restaurantes $(9.8 \%)$, en fiestas escolares $(9.3 \%)$ y en coches $(8.6 \%)$. También se les preguntó en dónde aceptaban sus padres que consumieran bebidas alcohólicas y los lugares donde más se los permiten es en su casa $(33 \%$ de los que beben más de cinco copas por ocasión y $29 \%$ de los que beben menos de esta cantidad) y en fiestas o reuniones sociales (42.7 y $19.9 \%$, respectivamente). Asimismo, se les cuestionó si sus padres les habían pedido que compraran, destaparan una botella o sirvieran una copa a sus familiares, y 21.7\% de los abstemios, 45.7\% de los que beben menos de cinco copas y $62.7 \%$ de los que beben mayores cantidades contestaron que sí.

\section{Drogas}

En el cuadro I se observa que $5.1 \%$ ha probado alguna droga en su vida. Un número más alto son hombres, tienen 18 años o más y tuvieron un empleo de medio tiempo en el año anterior a la encuesta; 3.7\% de estos usuarios cursaban la secundaria, y $8.4 \%$, la preparatoria.

Los inhalables $(2.3 \%)$ son los preferidos; en segundo lugar están tanto la mariguana $(1.7 \%)$ como los tranquilizantes $(1.7 \%)$ y $1.2 \%$ ha probado cocaína. Al igual que en encuestas anteriores con esta población, los resultados encontrados en este estudio indican que en las mujeres predomina el uso de tranquilizantes $(1.8 \%)$ y anfetaminas $(1.3 \%)$ sin receta médica, y los varones tienden a experimentar con drogas ilegales, principalmente con inhalables y mariguana $(2.7 \%$ en ambos casos).

Por otro lado, 3.7\% declaró que en los últimos 12 meses consumió alguna droga al mismo tiempo que bebió alcohol. El 14.4\% consideró que le sería fácil conseguir drogas si quisiera. Una alta proporción señaló que, si lo deseara, las conseguiría con extraños o desconocidos $(40.3 \%)$, con amigos o conocidos $(24.5 \%)$ o incluso con compañeros de la escuela (15.9\%).

\section{Variables relacionadas con el consumo de alcohol y drogas}

Se les cuestionó acerca de lo que acostumbran hacer en su tiempo libre (cuadro II), y se observó que los estudiantes que no beben alcohol ni consumen drogas practican más actividades como los deportes, ayudar en casa, hacer la tarea y salir de paseo con la familia, en comparación con los consumidores de drogas y de altas cantidades de alcohol, quienes con más frecuencia salen con los amigos, no hacen nada y se aburren o acostumbran ir a beber con amigos en sus ratos libres. 


\section{Cuadro I}

\section{Consumo de alcohol y drogas entre estudiantes de la ciudad de Pachuca, Hidalgo. México, 1996}

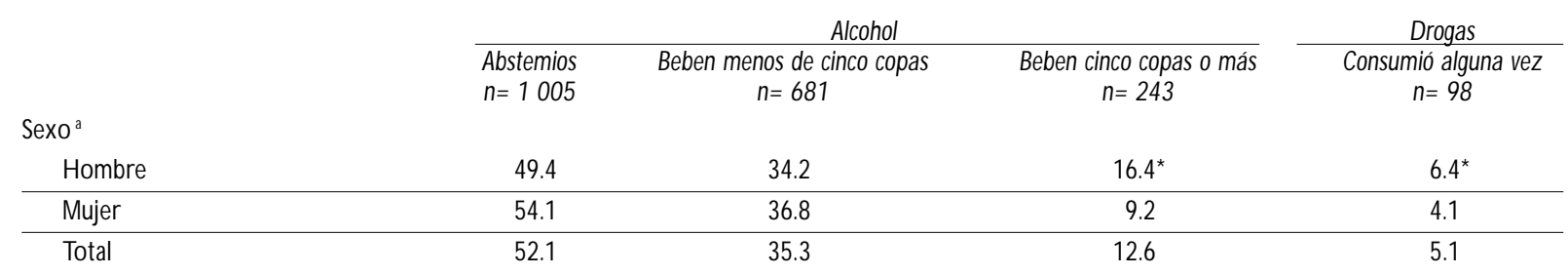

Edad ${ }^{b}$

\begin{tabular}{|c|c|c|c|c|}
\hline 130 menos & 70.4 & 25.3 & 4.2 & 2.4 \\
\hline 14 años & 47.0 & 39.9 & 13.1 & 4.5 \\
\hline 15 años & 37.5 & 45.2 & 17.3 & 9.0 \\
\hline 16 años & 33.1 & 41.6 & 25.3 & 7.9 \\
\hline 17 años & 21.8 & 55.1 & 23.1 & 6.4 \\
\hline 180 más & 29.0 & 34.8 & $36.2^{*}$ & $13.0^{*}$ \\
\hline
\end{tabular}

Estudió el año anterior ${ }^{b}$

\begin{tabular}{lllll} 
No estudió & 43.0 & 36.7 & $20.3^{*}$ & 6.3 \\
\hline Medio tiempo & 46.7 & 33.8 & 19.6 & 6.7 \\
\hline Tiempo completo & 53.3 & 35.5 & 11.2 & 4.7
\end{tabular}

Trabajó el año anterior ${ }^{b}$

\begin{tabular}{llllr} 
No trabajó & 52.9 & 35.9 & 11.2 & 4.5 \\
\hline Medio tiempo & 39.9 & 35.8 & $24.3^{*}$ & $12.2^{*}$ \\
\hline Tiempo completo & 50.0 & 29.5 & 20.5 & 5.7
\end{tabular}

$* p \leq 0.05$

N ota: para el consumo de alcohol las pruebas estadísticas utilizadas fueron la U de Mann W hitney (a) o Kruskall W allis (b). Para el consumo de drogas la prueba estadística utilizada fue $\chi^{2}$.

\section{Cuadro II \\ TIEMPO LIBRE Y CONSUMO DE ALCOHOL Y DROGAS ENTRE ESTUDIANTES de la ciudad de Pachuca, Hidalgo. México, 1996}

\begin{tabular}{|c|c|c|c|c|c|}
\hline \multirow[b]{2}{*}{ Actividades que realizan en su tiempo libre* } & \multicolumn{3}{|c|}{ Alcohol } & \multicolumn{2}{|c|}{ Drogas } \\
\hline & $\begin{array}{l}\text { Abstemios } \\
n=1005\end{array}$ & $\begin{array}{l}\text { Beben menos de cinco copas } \\
\qquad n=681\end{array}$ & $\begin{array}{l}\text { Beben cinco copas o más } \\
\qquad n=243\end{array}$ & $\begin{array}{c}\text { No usuarios } \\
n=1831\end{array}$ & $\begin{array}{c}\text { Usuarios } \\
n=98\end{array}$ \\
\hline Practica deporte & $78.5^{\ddagger}$ & 74.5 & 69.2 & $76.6^{\ddagger}$ & 63.9 \\
\hline Juega o sale con amigos & 68.9 & 77.8 & $86^{\ddagger}$ & 74.1 & 76.5 \\
\hline No hace nada y se aburre & 13.8 & 10.2 & 11.8 & 12.0 & $18^{\ddagger}$ \\
\hline Ayuda en las labores de la casa & $89.4^{\ddagger}$ & 76.4 & 64.7 & $82.6^{\ddagger}$ & 67.0 \\
\hline Hace tarea 0 estudia & $92.2^{\ddagger}$ & 84.4 & 74.1 & $87.8^{\ddagger}$ & 75.8 \\
\hline Juega $\mathrm{N}$ intendo o va a las maquinitas & 22.3 & 24.4 & 29.1 & 23.6 & 28.1 \\
\hline Sale a pasear con la familia & $74.6^{\ddagger}$ & 66.5 & 55.4 & $70.5^{\ddagger}$ & 48.9 \\
\hline Se va a beber con amigos & - & 10.0 & $40.5^{\ddagger}$ & 12.7 & $35.8^{\ddagger}$ \\
\hline
\end{tabular}

* La prueba estadística utilizada fue $\chi^{2}$

$\neq p \leq 0.05$ 
$\mathrm{Al}$ preguntarles acerca de las razones por las que consumen alcohol, los dos grupos de bebedores señalaron, en primer lugar, las siguientes: "Beber es una buena forma de celebrar" y "Bebo porque me gusta beber". Aproximadamente la tercera parte de los que beben mayores cantidades y alrededor de $15 \%$ de los que toman menos de cinco copas mencionaron: "es lo que hacen mis amigos cuando estamos juntos", "me ayuda a olvidarme de mis problemas y preocupaciones", "bebo cuando me siento tenso y nervioso" y "me da más confianza en mí mismo".

Por otra parte, ambos grupos de bebedores y, en especial, un número mayor de los que toman más alcohol, indicaron como principales problemas relacionados con su consumo los físicos tales como: "tener una cruda" (73.1 y 22.8\%) o "enfermarse del estómago" (44.4 y 15.8\%), "problemas con los padres" (41.6 y 10\%), "arrepentirse de algo que hizo al beber" (39.5 y 10.1\%) y "no recordar lo que hizo" (36.7 y 2.9\%).

Respecto a los resultados de la escala de actos antisociales (cuadro III), éstos indican que una gran parte de los bebedores altos, así como de los consumidores de drogas, tienen conductas tales como: tomar parte en riñas, dañar bienes ajenos, portar armas para defenderse, pasar la noche fuera de su casa sin permiso y tomar mercancía de tiendas sin pagarla.
En lo que se refiere a las normas familiares (cuadro IV), se observan grandes diferencias, ya que un porcentaje significativamente superior de los abstemios, en contraste con los que beben altas cantidades o consumen drogas, cumplen con las reglas que tienen sus padres respecto a las bebidas alcohólicas y, a su vez, les interesa más hacerlo. Es importante subrayar que una cifra más elevada de los no usuarios señaló que sus padres se darían cuenta si bebieran alcohol, y el único reactivo en el cual no hubo diferencias entre los grupos es el que se refiere a la claridad en las reglas sobre el consumo.

En la sección de conflictos familiares, una proporción significativamente mayor de los adolescentes que han probado drogas (37.1\%) y de los que beben más $(30.7 \%)$ informaron que es común que sus padres peleen más frecuentemente, consideran esta forma de pelear exagerada; en esos problemas ha intervenido el consumo de alcohol. También un número mayor mencionó que han pedido ayuda por estos conflictos y a su vez han intentado separarse, en contraste con aquellos que nunca han consumido alcohol o drogas.

Por otro lado, en el cuadro $V$ puede observarse que una cifra estadísticamente más elevada de los que beben mayores cantidades, señaló que tanto sus parientes como su mejor amigo frecuentemente ingieren alcohol. En el caso de los que han consumido drogas, los porcentajes

\section{Cuadro III \\ ACtOS ANTISOCIALES Y CONSUMO DE ALCOHOL Y DROGAS ENTRE ESTUDIANTES de la ciudad de Pachuca, Hidalgo. México, 1996}

\begin{tabular}{|c|c|c|c|c|c|}
\hline \multirow[b]{2}{*}{ Actos antisociales* } & \multicolumn{3}{|c|}{ Alcohol } & \multicolumn{2}{|c|}{ Drogas } \\
\hline & $\begin{array}{l}\text { Abstemios } \\
n=1005\end{array}$ & $\begin{array}{l}\text { Beben menos de cinco copas } \\
\qquad n=681\end{array}$ & $\begin{array}{l}\text { Beben cinco copas o más } \\
\qquad n=243\end{array}$ & $\begin{array}{c}\text { No usuarios } \\
n=1831\end{array}$ & $\begin{array}{l}\text { Usuarios } \\
n=98\end{array}$ \\
\hline Portar objeto filoso para defensa en caso de pelea & 4.9 & 9.7 & $21.7^{\ddagger}$ & 8.0 & $21.6^{\ddagger}$ \\
\hline Atacar a alguien con arma & 1.8 & 2.4 & $11.7^{\ddagger}$ & 2.5 & $17.2^{\ddagger}$ \\
\hline Manifestar crueldad con animales & 1.9 & 4.4 & $11.2^{\ddagger}$ & 3.4 & 14.0 \\
\hline Pasar la noche fuera de casa sin permiso & 1.6 & 5.8 & $21.6^{\ddagger}$ & 4.7 & $21.8^{\ddagger}$ \\
\hline Tomar auto sin permiso del dueño & 0.6 & 2.0 & $12.9^{\ddagger}$ & 2.0 & 16.1 \\
\hline Dañar ajenos & 2.0 & 6.8 & $18.1^{\ddagger}$ & 4.8 & $24.4^{\ddagger}$ \\
\hline Vender drogas & 0.1 & 0.6 & 6.6 & 0.7 & $8.8^{\ddagger}$ \\
\hline Tomar dinero o cosas que no le pertenecen (50 pesos) & 1.7 & 4.8 & $11.9^{\ddagger}$ & 3.5 & $14.3^{\ddagger}$ \\
\hline Tomar dinero o cosas que no le pertenecen (500 pesos) & 0 & 0.8 & 4.5 & 0.6 & $5.7^{\ddagger}$ \\
\hline Golpear o herir a alguien & 1.8 & 3.9 & $12.5^{\ddagger}$ & 3.5 & $11.2^{\ddagger}$ \\
\hline Forzar cerraduras & 0.2 & 1.5 & 5.3 & 1.1 & 5.5 \\
\hline Tomar parte en riñas & 1.0 & 4.8 & $21.8^{\ddagger}$ & 4.2 & $19.1^{\ddagger}$ \\
\hline Prender fuego a bienes ajenos & 0.3 & 1.9 & 8.8 & 1.4 & $11.0^{\ddagger}$ \\
\hline Golpear o herir a autoridad & 0.2 & 0.9 & 4.0 & 0.8 & $4.4^{\ddagger}$ \\
\hline Tomar mercancía de tienda sin pagarla & 1.6 & 6.2 & $20.8^{\ddagger}$ & 5.0 & $18.2^{\ddagger}$ \\
\hline Amenazar a persona con arma para obtener algo & 0.2 & 1.4 & 2.2 & 0.7 & 4.4 \\
\hline
\end{tabular}

*La prueba estadística utilizada fue $\chi^{2}$

$\neq p \leq 0.05$ 


\section{Cuadro IV \\ Normas familiaRes Y CONSUMO DE ALCOHOL Y DROGAS ENTRE ESTUdianteS de la ciudad de Pachuca, Hidalgo. México, 1996}

\begin{tabular}{|c|c|c|c|c|c|c|}
\hline \multirow[b]{2}{*}{ Normas familiares* } & \multicolumn{4}{|c|}{ Alcohol } & \multicolumn{2}{|c|}{ Drogas } \\
\hline & $\begin{array}{l}\text { Abstemios } \\
n=1005\end{array}$ & $\begin{array}{l}\text { Beben menos de cinco copas } \\
\qquad n=681\end{array}$ & $\begin{array}{l}\text { Beben cinco copas o más } \\
n=243\end{array}$ & $\begin{array}{c}\text { Total } \\
n=1929\end{array}$ & $\begin{array}{l}\text { No usuarios } \\
n=1831\end{array}$ & $\begin{array}{c}\text { Usuarios } \\
n=98\end{array}$ \\
\hline Siempre cumple las reglas de sus padres & $77.4^{\ddagger}$ & 66.4 & 51.5 & 70.2 & $71.4^{\ddagger}$ & 49.0 \\
\hline \multicolumn{7}{|l|}{ Sus padres tienen una norma estricta } \\
\hline que no debe tomar bebidas alcohólicas & $78^{\ddagger}$ & 67.6 & 47.2 & 70.5 & $71.4^{\ddagger}$ & 53.8 \\
\hline Sus padres se darían cuenta si bebiera alcohol & $87.0^{\ddagger}$ & 80.6 & 64.9 & 82.0 & 83.0 & 62.6 \\
\hline \multicolumn{7}{|l|}{ Sus padres le han puesto las reglas muy claras sobre } \\
\hline su forma de beber & 62.0 & 66.8 & 61.6 & 63.7 & 64.1 & 56.7 \\
\hline \multicolumn{7}{|l|}{ Es importante para él/ella: } \\
\hline Hacer siempre lo que sus padres le dicen que haga & $83.5^{\ddagger}$ & 79.9 & 62.2 & 79.5 & $80.3^{\ddagger}$ & 65.3 \\
\hline \multicolumn{7}{|l|}{ Cumplir las normas de sus padres acerca } \\
\hline de beber alcohol & 80.9 & $84.8^{\ddagger}$ & 64.7 & 80.2 & $81.1^{\ddagger}$ & 63.8 \\
\hline
\end{tabular}

* La prueba estadística utilizada fue $\mathrm{X}^{2}$

$\neq p \leq 0.05$

que resultan ser significativos únicamente se ubican en el padre y en su mejor amigo.

\section{Discusión}

Los datos sobre la prevalencia alguna vez en la vida de consumo de alcohol, obtenidos en los estudiantes de enseñanza media y media superior de la ciudad de Pachuca, Hidalgo, son similares a los encontrados en toda la entidad en la encuesta nacional estudiantil de 1991 ( 47.9 y $41 \%$, respectivamente). Sin embargo, no sucedió lo mismo con los que bebieron en el mes anterior, ya que la prevalencia en este estudio fue muy superior (20.9\%) en comparación con lo encontrado en 1991 (10.1\%). A pesar de que los datos de estas dos investigaciones no son totalmente comparables debido a que los resultados de 1991 pertenecen a todo el estado de Hidalgo y los de 1996 son representativos de la ciudad de Pachuca, ambas encuestas abarcaron a la población escolar. Por lo tanto, se puede señalar que el número de jóvenes que han probado las bebidas alcohólicas se ha mantenido más o menos estable; no obstante, en 1996 se detectaron más usuarios activos al momento del estudio en esta población, aspecto que refleja una mayor concentración de usuarios en escenarios urbanos y un aumento en el número total de consumidores, como se ha observado en otras regiones. Por ejemplo, en el Distrito Federal en tres mediciones con esta población en
1989, 1991 y 1993 el consumo en el mes anterior se elevó de $15 \%$ en 1989 a $24 \%$ en $1993 .{ }^{31}$

Respecto al consumo de drogas ilegales y médicas usadas fuera de prescripción, el número de consumidores experimentales se ha mantenido más o menos constante de 1991 a 1996, en relación con las siguientes sustancias: tranquilizantes $1.5 \%$ en 1991 y $1.7 \%$ en 1996; mariguana 0.9 y $1.7 \%$; y cocaína 1.1 y $1.2 \%$. Únicamente en el caso de los inhalables, que han sido las drogas de preferencia, pareciera que ha disminuido el número de usuarios, pues fue de $5.7 \%$ en 1991 y de $2.3 \%$ en 1996.

Una proporción mayor de los estudiantes que ingirieron grandes cantidades de bebidas alcohólicas durante el mes anterior y de los que han probado drogas, en contraste con los adolescentes que nunca han tomado alcohol y con los que beben cantidades moderadas, presentan un perfil que coincide con el observado en las encuestas estudiantiles precedentes, $, 10,13,16,20$ a saber: son varones, tienen más edad y están inmersos en el mundo adulto, puesto que tienen la responsabilidad de un trabajo que a veces combinan con los estudios.

Por otra parte en México, al igual que en otros países, existe una regulación que prohibe la venta de alcohol a menores de edad, misma que en muchas ocasiones no se cumple en los lugares donde se vende y distribuye. Esto se refleja en los datos, ya que los ado- 


\section{Cuadro V \\ Consumo frecuente de alcohol en familiares y amigos, entre estudiantes de la ciudad de Pachuca, Hidalgo. México, 1996}

\begin{tabular}{|c|c|c|c|c|c|c|}
\hline \multirow[b]{2}{*}{ Consumo frecuente de alcohol } & \multicolumn{4}{|c|}{ Alcohol* } & \multicolumn{2}{|c|}{ Drogas ${ }^{\ddagger}$} \\
\hline & $\begin{array}{l}\text { Abstemios } \\
n=1005\end{array}$ & $\begin{array}{l}\text { Beben menos de cinco copas } \\
\qquad n=681\end{array}$ & $\begin{array}{l}\text { Beben cinco copas o más } \\
\qquad n=243\end{array}$ & $\begin{array}{c}\text { Total } \\
n=1929\end{array}$ & $\begin{array}{c}\text { No usuarios } \\
n=1831\end{array}$ & $\begin{array}{c}\text { Usuarios } \\
n=98\end{array}$ \\
\hline Padre & 6.9 & 11.8 & $18.5^{\S}$ & 10.1 & 9.6 & $20.4^{\S}$ \\
\hline Madre & 1.4 & 2.8 & $4.6^{5}$ & 2.3 & 2.0 & 7.4 \\
\hline Hermanos & 2.0 & 4.2 & $12.1^{\S}$ & 4.1 & 3.6 & $12.8^{5}$ \\
\hline o tros parientes & 14.9 & 20.6 & $24.1^{5}$ & 18.1 & 17.7 & 25.0 \\
\hline Su mejor amigo & 4.5 & 6.9 & $21.7^{\S}$ & 7.5 & 6.7 & $21.9^{5}$ \\
\hline
\end{tabular}

* La prueba estadística utilizada fue Kruskall W allis

₹ La prueba estadística utilizada fue U de Mann W hitney

${ }^{\S} p \leq 0.05$

lescentes mencionaron que obtienen bebidas alcohólicas principalmente en tiendas donde no les piden identificación.

Respecto al tipo de bebida, la literatura y en general los estudios desarrollados en México ${ }^{3,8}$ indican que predomina el consumo de cerveza; asociado a esto, en los últimos años ha destacado el consumo de bebidas como los coolers, que han tenido una gran aceptación entre los jóvenes; en la encuesta levantada en 1993 con alumnos del Distrito Federal, ${ }^{29}$ especialmente entre las mujeres $^{32}$ y en específico en la presente encuesta, manifestaron que este tipo de bebidas, al igual que la cerveza, son las que más acostumbran tomar. Esto conduce a reflexionar sobre la información que están recibiendo ya que pareciera que la publicidad y, en general, la idea comercial que gira en torno a la venta de estas bebidas, está principalmente dirigida a los jóvenes.

Es interesante observar que, en una alta proporción de casos, estos jóvenes toman bebidas alcohólicas en sus hogares, lo cual coincide con diferentes investigaciones realizadas tanto en población adulta ${ }^{33,34}$ como entre estudiantes. ${ }^{35,36}$ Aunado a lo anterior, declararon que este consumo se presenta con la aceptación parental; especialmente un número mayor de los que beben altas cantidades han tenido contacto con el alcohol por medio de sus padres, lo que sugiere que existe un ambiente que favorece el consumo de bebidas alcohólicas al haber cierta tolerancia social hacia esta conducta, ya que en diferentes núcleos sociales, como la propia familia y el grupo de amigos, forman parte de las celebraciones y festividades cotidianas de estos estudiantes, quienes en su mayoría son menores de edad.

Por otra parte, las actividades a las que se dedican en su tiempo libre reflejan variaciones importantes entre los abstemios, en contraste con los que beben más de cinco copas y con los consumidores de drogas, quienes informan no hacer nada y aburrirse con más frecuencia. $\mathrm{Al}$ respecto De la Fuente ${ }^{2}$ menciona que el entorno social de los jóvenes les ofrece limitadas oportunidades de desarrollo educativo, ocupacional y del uso del tiempo libre. Esto subraya la necesidad de que en la familia, así como en la escuela, se fomenten actividades culturales, deportivas y recreativas que fortalezcan y apoyen los intereses e inquietudes propios de su edad.

Los resultados de esta encuesta corroboran los encontrados en mediciones anteriores en esta población, debido a que ingerir elevadas cantidades de alcohol, así como consumir tanto drogas ilegales como médicas, está muy asociado con incurrir en conductas antisociales $^{15-18}$ que ponen en riesgo su integridad física, moral y legal.

Otro hecho que sobresale es que los alumnos que consumen tanto drogas como cantidades importantes de bebidas alcohólicas parecen estar más distanciados de sus familias, puesto que salen menos a pasear juntos y ayudan menos en las labores de su casa. Del mismo modo, un número inferior cumple con las normas paternales y muestran un reducido interés en apegarse a las mismas. Cabe destacar que independientemente de si lo han consumido o no, una gran parte (40\%) manifestó que dentro de su núcleo familiar no existen reglas claras hacia el consumo de alcohol, aspecto que subraya la importancia que tienen los padres en definir abiertamente los parámetros que guíen las acciones de sus hijos ya que, a esta temprana edad, cualquiera puede ser vulnerable en cuanto a incurrir en conductas socialmente inaceptables.

En lo que se refiere a la interacción familiar, los datos del estudio corroboran lo encontrado en otras encuestas $^{21-23} y$, en especial, lo mencionado por Maltzman 
y Schweiger, ${ }^{26}$ ya que los alumnos que no han consumido drogas o alcohol perciben más funcional su ambiente familiar, debido a que declararon tener menos conflictos e intentos de separación dentro de sus familias. Asimismo, tanto los consumidores de drogas como los que toman más bebidas alcohólicas informaron que, en su entorno social, sus amigos y parientes consumen frecuentemente alcohol, lo que coincide con algunos estudios $^{19-22}$ y confirma que estos jóvenes están expuestos a un riesgo superior;, ${ }^{12,13,20}$ asimismo, subraya la trascendencia que tiene la familia en el establecimiento de modelos de comportamiento para los hijos.

En resumen, los datos señalan que una proporción importante de los menores bebe alcohol y prefiere bebidas como la cerveza o aquellas que la publicidad ha dirigido hacia este sector del mercado (coolers y otras bebidas preparadas), contando para ello con la anuencia de los padres de familia y de las autoridades, quienes no cumplen la norma de prohibir su venta a los menores de edad.

Los estudiantes que beben grandes cantidades y los que consumen drogas, presentan más consecuencias derivadas del uso de sustancias, más problemas de conducta, con menos frecuencia hacen deporte o comparten actividades con sus familias, a la vez que perciben un mayor índice de conflictos dentro de ellas $\mathrm{y}$ viven en ambientes en donde se bebe más alcohol.

Puede concluirse que el consumo de alcohol está normalizado en la población adolescente; esta observación debe replantear los objetivos de los programas de prevención, dado que no existe un consenso en cuanto a que los menores de edad no deben beber. Podría considerarse la realización de una campaña orientada a los adultos que están cerca de los menores y, específicamente, a los padres, sobre los efectos nocivos a la salud del consumo de alcohol, razones que apoyarían la meta de retrasar el inicio en el consumo, o bien, de orientar los programas a la educación en el consumo responsable del alcohol, así como hacia la disminución de riesgos. En cualquier caso, es necesario informar a los padres de familia sobre la influencia que sus prácticas de consumo tienen en los menores, promover actividades que los lleven a una mejor calidad de vida, disminuir los riesgos e identificar a aquellos adolescentes con mayor tendencia a abusar en su consumo de alcohol y de drogas, debido a las características del ambiente en donde se desenvuelven, con el fin de apoyarlos mediante intervenciones preventivas más intensas.

Los datos obtenidos en esta investigación proporcionan una amplia información sobre la epidemiología del uso de sustancias en la población estudiantil de la ciudad de Pachuca, Hidalgo, por lo que, con base en estos hallazgos, la comunidad ha empezado a efectuar diversas acciones preventivas tales como la realización de programas informativos, en los diferentes medios de comunicación, sobre el consumo de alcohol y sus consecuencias. Asimismo, se han impartido conferencias en las que se ha retroalimentado e informado tanto a la comunidad escolar-directivos y maestros-, como a los padres de familia y jóvenes; se ha publicado también un boletín informativo que contiene los resultados de esta investigación. Adicionalmente los profesores han desarrollado distintas actividades preventivas con sus alumnos.

Por último, cabe destacar la misión conjunta que tienen la sociedad, los medios de comunicación, las autoridades, los padres de familia y el sector educativo, para seguir coordinando esfuerzos y estrategias preventivas permanentes -como las realizadas con la población escolar de Pachuca, Hidalgo, a fin de enfrentar este fenómeno tan complejo-, que impidan que los jóvenes se involucren en el consumo de drogas y que ingieran cantidades excesivas de bebidas alcohólicas, que les ocasionen problemas severos.

\section{Agradecimientos}

Se agradece el apoyo incondicional proporcionado tanto por el Consejo Estatal como por el Consejo $\mathrm{Mu}-$ nicipal contra las Adicciones, del estado de Hidalgo.

\section{Referencias}

1. Medina-Mora ME,Tapia R, Rascón ML, Solache G, 0 tero B, Lazcano F et al. Situación epidemiológica del abuso de drogas en México. Bull Sanit Panam 1989;107:475-484.

2. De la Fuente R, Medina-Mora ME, C araveo J. Salud mental en México. México, D. F.: Instituto Mexicano de Psiquiatría/Fondo de Cultura Económica, 1997:183-201.

3. Medina-Mora ME,Tapia R, Sepúlveda J, Rascón ML, Mariño MC,Villatoro J. Los patrones del consumo de alcohol y los síntomas de dependencia en la población urbana de la República Mexicana. Anales. Reseña de la VI Reunión de Investigación. México, D. F.: Instituto Mexicano de Psiquiatría, 1991:133-137.

4. Borges G, Kershenobich D, Pelcastre B, Gil A, Inda M, Garrido F. Cirrosis hepática y consumo de alcohol en un estudio colaborativo: Instituto Mexicano de Psiquiatría-Instituto $\mathrm{N}$ acional de la $\mathrm{N}$ utrición.A nales. Reseña de la VII Reunión de Investigación. México, D. F.: Instituto Mexicano de Psiquiatría, 1992:130-138.

5. Secretaría de Salud-Dirección General de Estadística e Informática. Mortalidad 1995. México, D. F.: SSA, 1996.

6. Medina-Mora ME,Tapia R, 0 tero B, Rascón ML, Mariño MC,Villatoro J et al. Factores asociados con el inicio en el consumo de drogas. Resultados de una muestra nacional. La psicología social en México. México, D. F.: Asociación Mexicana de Psicología Social, 1990;3:214-218. 
7.Tapia-C onyer R, Medina-Mora ME, Sepúlveda J, D e la Fuente R, Kumate J. La encuesta nacional de adicciones de México. Salud Publica Mex 1990;32(5):507-522.

8.Tapia-C onyer R, C ravioto P, D e la Rosa B, Kuri P.Panorama epidemiológico de las adicciones 1993. En: Situación actual de las adicciones en México. México, D.F.: SSA-CO N ADIC, 1995:19-25.

9. Castro ME, Maya MA. El consumo de alcohol en la población estudiantil. Salud Mental 1987;10:52-58.

10. Medina-Mora ME, Rojas E, Juárez F, Berenzon S, C arreño S, G alván J et al. Consumo de sustancias con efectos psicotrópicos en la población estudiantil de enseñanza media y media superior de la República Mexicana. Salud Mental 1993;16:2-8.

11. Rojas E, Medina-Mora ME, Juárez F,C arreño S,Villatoro J, Berenzon S et al. El consumo de bebidas alcohólicas y variables asociadas entre los estudiantes de México. Salud Mental 1995;18:22-27.

12. De la Fuente R. La patología mental y su terapéutica. México, D. F.: Fondo de Cultura Económica, 1997; vol .1:135-157.

13. Medina-Mora ME, Mariño MC , Berenzon S, Juárez F, C arreño S. Factores asociados con la experimentación y con el uso problemático de drogas. Las adicciones en México: hacia un enfoque multidisciplinario. México, D.F.:SSA-CONADIC, 1992:87-97.

14.Villatoro J, Fleiz C, Rivera E, Medina-Mora ME. La relación del ambiente familiar y el grupo de pares en el consumo de drogas del adolescente. Congreso Regional de Psicología para Profesionales en América: Entrelazando la Ciencia y la Práctica en Psicología;1997 27 julio -2 agosto, México, D.F.

15. C astro ME, Rojas E, García G, De la Serna J. Epidemiología del uso de drogas en la población estudiantil.Tendencias en los últimos 10 años. Salud Mental 1986;9:80-86.

16. C astro ME, G arcía G, Rojas E, De la Serna J. C onducta antisocial y uso de drogas en una muestra nacional de estudiantes mexicanos. Salud Publica Mex 1988:30:216-226.

17. Berenzon S, López E, Medina-Mora ME,Villatoro J, Juárez F, C arreño S et al. Uso de inhalables entre estudiantes y condiciones relacionadas al consumo: actos antisociales. La psicología social en México. México, D. F.: Asociación Mexicana de Psicología Social, 1994;5:351-358.

18. Juárez F, Berenzon S, Medina-Mora ME,Villatoro J, C arreño S, López E et al.Antisocial behavior: Its relation to selected sociodemographic variables and alcohol and drug use among Mexican students. Subst Use Misuse 1998:33:1441-1463.

19.Villatoro J, Medina-Mora ME, Fleiz C, Juárez F, Berenzon S, López E et al. Factores que predicen el consumo de drogas en los estudiantes de enseñanza media y media superior de México. La psicología social en México. Hermosillo, Son.: Asociación Mexicana de Psicología Social, 1996;6:569-574

20. Dielman TE, Butchart AT, Shope JT, Miller M. Environmental correlates of adolescent substance use and misuse: Implications for prevention programs. Int J Addict 1990-1991;25(7A-8A):855-880.
21. Madianos MG, Madianos D, Stefanis C. Familial correlates of drug and alcohol use in a nationwide general population survey. Psychopathology 1995;28:85-94.

22. Muetzell S. Mixed abuse of alcohol and drugs in teenagers in Sweden vs a group from the general population. Early Child Dev Care 1995;113: 93-105.

23. C astro ME, 0 rtiz A, C audillo $C, C$ hávez AM. Alteraciones cognitivas y problemática psicosocial asociadas con el consumo de drogas. Salud Mental 1985:8:74-79

24. Pons ], Berjano $E$, G arcía, F.Variables que discriminan el consumo abusivo de alcohol en la adolescencia. Adicciones 1996;8:177-191.

25. Denton RE, Kampfe CM.The relationship between family variables and adolescent susbtance abuse: A literature review. Adolescence 1994; 29: 475-495.

26. Maltzman I, Schweiger A. Individual and family characteristics of middle class adolescents hospitalized for alco hol and other drug abuse. Br JAddict 1991;86(11):1435-1447.

27. Pons ], Berjano E. El inicio en el consumo de drogas en relación a las dimensiones del autoconcepto en adolescentes. Rev Esp Drogodepend 1996:21:229-244.

28. Furlong A, C artmel F.Young people and social change. Individualization and risk in late modernity. Buckingham: 0 pen University Press,1997.

29. Berenzon S, Medina-Mora ME, C arreño S, Juárez F,Villatoro J, Rojas E. Las tendencias del consumo de sustancias psicoactivas entre estudiantes de enseñanza media y media superior en el Distrito Federal, 1993. Salud Mental 1996;19:1-5.

30. Medina-Mora ME, Gómez-Mont F, Campillo-Serrano C. Validity and reliability of a high school drug use questionnaire among Mexican students. Bull N arc 1981;33:67-76.

31. Rojas E, Medina-Mora ME,Villatoro J, Juárez F, C arreño S, Berenzon S. Evolución del consumo de drogas entre estudiantes del Distrito Federal. Salud Mental 1998:21:37-42.

32. Berenzon S, C arreño S, Medina-Mora ME, Juárez F,Villatoro J, Rojas E. Prácticas del uso de alcohol entre jóvenes estudiantes de la ciudad de México.Variaciones por género. Cultura Psicológica 1994:3:23-25.

33. Campillo C, Díaz R, Romero M, Padilla P. El médico general frente al bebedor problema. Salud Mental 1988;11:4-12.

34. Medina-Mora ME. Factores sociales relacionados con el consumo de alcohol en México y Estados Unidos. Anales. Reseña de la II Reunión de Investigación. México, D. F.: Instituto Mexicano de Psiquiatría, 1984:181193.

35. Maya MA, García G. Estudio epidemiológico sobre el consumo de alcohol en población joven de 14 a 18 años. Salud Publica Mex 1986;28: 371-379.

36. Mas C, Ramírez MA. Consumo de alcohol y drogas en una población de jóvenes estudiantes y no estudiantes. Salud Mental 1985;8:71-73. 